\title{
Multiscale Spectral Methods for Data Indexing in a Radon Space
}

\section{Petrov}

Key Words: Computed tomography images; CBIR-system; Wavelet Transform; Shearlet Transform.

\begin{abstract}
This paper presents three methods for multiscale indexing of the content of projection data of computed tomography images in the CBIR-systems for medical database search. The feature spaces in the developed algorithms have been created by means of, respectively: Discrete Stationary Wavelet Transform (DSWT), Shearlet Transform (ST) and Repagulum Wavelet Transform (RWT). A comparative analysis and assessment of the proposed algorithms have been carried out based on experimental studies with computed tomography images.
\end{abstract}

\section{Introduction}

One of the most common non-invasive imaging techniques used in modern medicine is computed tomography $(C T)$. It is characterized by a high level of informativeness, reliability and efficiency which allows registering even minor pathological changes in the structure of the analyzed organ. $C T$ performs simultaneous visualization of tissue structures of different density - bone structures, soft tissue structures and blood vessels. The early, timely and correct diagnosis is the basis for enhancing the efficacy of the planned treatment methods for a specific disease.

$C T$ is a method for obtaining a detailed $2 D$ image of anatomical sections obtained by means of computer processing of datasets specified by the intensity of the $\mathrm{X}$-ray radiation that has penetrated to a certain depth through a thin layer of tissues in the human body. $C T$ images are obtained after their successive passing through the phases of scanning (obtaining projection data), reconstruction (recovery) and visualization.

In modern systems for archiving and sharing images - PACS (Picture Archiving and Communication System), patient studies are accessed using various criteria: identification number, patient's name, date or period of the study and description of the study. In connection with the clinical diagnostic practice in decision making, it is often necessary to find images of the same modality, anatomical region or pathology. That is why medical database-oriented CBIR-systems (Content Based Image Retrieval) have been actively developed in recent years, although part of the necessary information is contained in the header portion of the DICOM (Digital Imaging and Communications in Medicine) images [1].

According to a basic principle in the decision-making statistical theory, each pre-processing of raw data can only reduce, but not increase, the information they contain [2]. Therefore, information would be most efficiently applied if the respective analysis is conducted directly using projection data, not a restored tomography image.

The present work considers searching systems that use large-scale indexing of the content of projection data of $C T$ images. The feature spaces in these systems have been built by: Discrete Stationary Wavelet Transform (DSWT), Shearlet Transform (ST) and Repagulum Wavelet Transform (RWT).

\section{Multiscale Transformations}

When analyzing and processing non-stationary or nonuniform data, the necessity of locating the spatial coordinates of the signal consistent with its specific frequencies (scales) requires substituting the Fourier transform with the wavelet transform. Their presentation by means of wavelet-systems is associated with different levels of permissions (resolutions) and localization. Let $L_{2}(R)$ be the space of limited-energy signals. Scaling (dilation) and translation operators applied to the so-called generating function $\psi \in L_{2}(R)$ or wavelet underlie the building of these systems [3]. The discrete wavelet-system in $L_{2}(R)$ is defined by the set $W_{\psi}=\left\{\psi_{j, m}=D_{2}^{-1} T_{m} \psi=2^{j / 2} \psi\left(2^{j} \bullet-m\right), \quad j, m \in Z\right\}$, where $D_{2} \psi(x)=2^{-1 / 2} \psi\left(2^{-1} x\right)$ and $T_{t} \psi(x)=\psi(x-t), t \in R$. The discrete wavelet-transform associated with this system is defined by the scalar product $W_{\psi} f(j, m)=\left\langle f, \psi_{j, m}\right\rangle, f \in L_{2}(R)$.

In spite of their advantages in signal and image processing tasks, wavelets are not very efficient in processing multidimensional data. A significant disadvantage of wavelet-systems is their limitation in the study of, for example, anisotropic singularities of a given image. These studies require the elements of analyzing systems to vary over several scale levels, to be oriented and to be able to get very elongated at times [4]. Shearlet systems can achieve this after the introduction of a special scaling operator $D_{A_{u}}$ (generating elements in different scales), a shearing operator (in charge of orientation) and a translating operator. The $D_{A_{a}}\left(a \in R^{+}\right)$operator is most commonly determined by the parabolic scaling matrix $A_{a}=\operatorname{diag}(a, \sqrt{a})$, and $D_{S_{s}}(s \in R)$ by using the shearing matrix $S_{s}=\left(\begin{array}{ll}1 & s \\ 0 & 1\end{array}\right)$.

For $\psi \in L_{2}(R)$, the continuous shearlet-system $s h_{\psi}$ is defined by $s h_{\psi}=\left\{\psi_{a, s, t}=T_{t} D_{S_{s}} D_{A_{a}} \psi, a>0, s \in R, t \in R^{2}\right\}$, and its shearlet-transformation of $f \in L_{2}\left(R^{2}\right)$ $s h_{\psi} f(a, s, t)=\left\langle f, \psi_{a, s, t}\right\rangle$. 
There are different algorithms for the realization of the discrete shearlet-transformation $[5,6]$. This study uses the Fast Finite Shearlet Transform algorithm, proposed by $S$. Hauser.

The third multi-scale transformation that has been studied is the $R W T$ [7] which uses a family of functions $\psi(x, a)$, localized at one point. The continuous $R W T f \in L_{2}(R)$ is defined by the convolution $W_{\psi}{ }^{r} f(a, b)=f(x) * \overline{\psi_{a}}(b)$, where $\overline{\psi_{a}}(x)=\psi(-x, a)$. The $a$ parameter characterizes the regularity (usually the Hölder's indicator is selected as such at the localization point) of the underlying repagulum wavelet, whereas $b$ sets the offset along the time axis and determines the convolution. When applying the $R W T$, the $\psi(x, a)=2^{-x a}$ function is often preferred as a basic wavelet because of the numerical implementation. Then $W_{\psi}{ }^{r} f(a, b)$ is obtained by convolution with the filter $\left\{G_{a}(n)\right\}_{n=0}^{2\left(N_{a}-1\right)}=\left\{-2^{-\left(N_{a}-1\right) a}, \ldots,-2^{-a},-1,1,2^{-a}, \ldots, 2^{-\left(N_{a}-1\right) a}\right\}$.

The value of the parameter of the $R W T$ can be set by means of the following formula $a=2^{-j} \alpha_{0}$, where $\alpha_{0}$ is a fixed number from the interval $(0,1)$, and $j=1,2,3, \ldots$. The change of $j$ results in modifying the regularity parameter. Therefore, the transition from one authorization level to another under the $R W T$ is performed by changing the parameter $a$.

\section{Algorithms of CBIR-systems}

In the implementation of algorithms for content-based image retrieval, this work uses four indexed databases of two types, corresponding to $S=\left\{s_{i}(n, m)\right\}_{i \in I \subset \mathbb{N}}$ images or their projections $S_{R}$ in the Radon space. Let $\Re$ be any of the entered multiscale transformations, i.e. $\Re \in\left\{W_{\psi}, s h_{\psi}, W_{\psi}^{r}\right\}$. The descriptor matrices used are of two types in the corresponding indexed databases: $D_{\psi}^{\Re}(s)=\left\{P_{J, m_{\jmath}}^{\Re}(s), L_{J, m_{J}}^{\Re h}(s)\right\}_{s \in S_{S_{n}}}$ and $D_{\psi}^{s h}(s)=\left\{P_{J, m_{J}}^{s h}(s), L_{J, m_{j}}^{s h}(s)\right\}_{s \in S}$, where $m_{J}$ is respectively the number of the smallest and largest ratios derived from the selected $J^{\text {th }}$ decomposition level of the $s$ image by the $\Re$ transform, and $P_{*}^{*}$ and $L_{*}^{*}$ are respectively the arrays containing the values and positions of these local minima and maxima. Similar descriptor matrices are also built for each test image $s_{0}$. The similarity of $D_{\psi}^{\Re}(s)$ and $D_{\psi}^{\Re h}\left(s_{0}\right)\left(D_{\psi}^{s h}(s)\right.$ and $\left.D_{\psi}^{s h}\left(s_{0}\right)\right)$ matrices is estimated by the distances between their corresponding blocks: $\rho_{L^{*}}^{i}=\min _{i \in I} \rho_{0}\left(L_{*}^{*}\left(s_{i}\right), L_{*}^{*}\left(s_{0}\right)\right)$ and $\rho_{P^{*}}^{i}=\max _{i \in I} \rho\left(P_{*}^{*}\left(s_{i}\right), P_{*}^{*}\left(s_{0}\right)\right)$, where $\rho_{0}$ is the equality relation in the set of real numbers and $\rho$ is the metric function.

The content-based search result in the corresponding database is a set of similar (relevant) images $R_{S}=\left\{s_{i}, i \in I_{0} \subset I\right\}$, if $\rho_{L_{*}^{*}}^{i} \geq 2 \lambda_{J}^{*}$ and $\rho_{P_{*}^{*}}^{i} \leq \varepsilon^{*}$, for each $i \in I_{0}$, where the $\lambda_{J}^{*}$ and $\varepsilon^{*}$ parameters are additionally set by the user.

\section{Analysis and Assessment of Content-Based Search Algorithms}

The present paper analyzes two types of tasks: recognition (identification) and relevant-image detection. Performance assessment of the proposed algorithms, as well as a comparative analysis showing the advantages of projection data indexing in comparison to slice recovery have been carried out. For this purpose, experimental studies using $C T$ images of the heart and blood vessels have been performed. From a randomly selected subfolder 100 slices have been generated, divided into individual groups, based on their visual similarity which is confirmed using metrics taking into account the structural similarity of the analyzed images SSIM (Structural Similarity Image Measure).

For system assessment, additional images have been created out of each slice, obtained by adding Gaussian noise having the following parameters: means $\mu=0$ and dispersion values $v=0.0005,0.00075,0.001,0.005,0.0075,0.01$, 0.05 and 0.1 . The full range of representatives for each of the set groups have been used as a query, relevant slices from the corresponding group being all slices, as well as those further generated at $v=0.0005,0.00075,0.001,0.005$, 0.0075 .

In projection data recognition, each slice is subjected to the Radon transformation at an angle variation from 0 to $360^{\circ}$, at the rate of $1^{\circ}$. Each of the obtained projections is exposed to $D S W T, S T$ and $R W T$ at predetermined parameters. After that characteristic vectors are formed. At the entrance of the search system all sections of the formed sample are successively submitted as a query and their degree of similarity (in metric) is determined.

In order to conduct the second part of the analysis of algorithms, image recovery is performed on the basis of the resulting projection data using the filtered back-projection method.

The following metrics are used to assess the performance of the investigated content-based search algorithms: for recognition accuracy - precision; for recognition completeness - recall; and the harmonic mean of precision and recall $-F_{m}$. Accuracy is calculated as the ratio of the relevant retrieved images that have been found to the total number and it characterizes the system's ability to extract only relevant data from the database. Completeness indicates the ability of the system to extract the data necessary to the user without taking into account irrelevant images found as a result of the query. The parameter $F_{m}$ is the harmonic mean of precision and recall and it characterizes the system in terms of both measures simultaneously. These metric functions are calculated for the three cases where items of characteristic vectors are obtained by $D S W T, S T$ and $R W T$.

Figure 1 shows the different stages through which each slice goes: original image a), projection data (sonograms) and reconstructed images (based on projection data and coefficients of the three multiscale 
a)

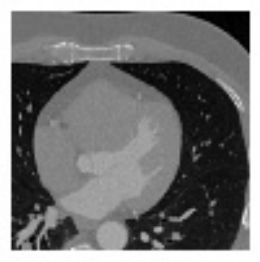

d)

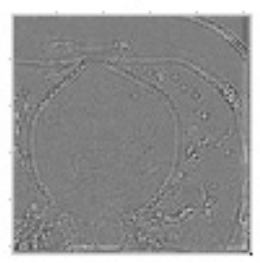

b)

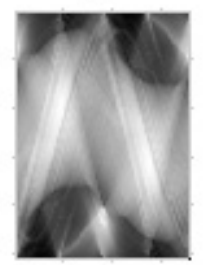

e)

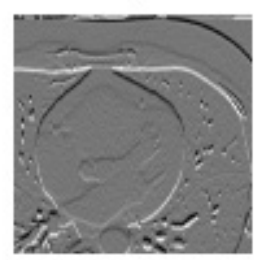

c)

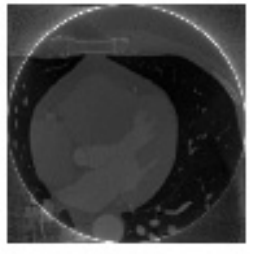

f)

Figure 1. Cardiac slice $(a)$, projections data $(b)$, reconstructed image: the filtered back-projection method $(c)$ and projections data $-D S W T, R W T$ and $S T(d \div f)$

transformations).

Figure 2 presents the results of the assessment of similarity (in metric) for system testing in the two experimental tasks: recognition (identification) and relevant-image detection. The results of the analysis assessing the quality of search algorithms are presented in Figure 3.

The obtained smaller similarity values when using the $S T$, compared to those of $D S W T$ and $R W T$, show that the shearlet algorithm has a higher degree of accuracy, even with a decreased descriptor dimension, which makes it preferable in tasks of extracting information from databases.
From the results presented in figure 3 it can be concluded that the search system using $S T$ is of superior quality compared to the systems based on DSWT and $R W T$ in terms of chosen assessment measures. This can be seen in the achievement of higher accuracy in both, extracting necessary data and finding relevant images of the submitted query.

Figure 4 shows the results of the second part of the analysis of recognition algorithms using projection data and reconstructed tomographic slices.

The resulting data show the higher efficiency of using

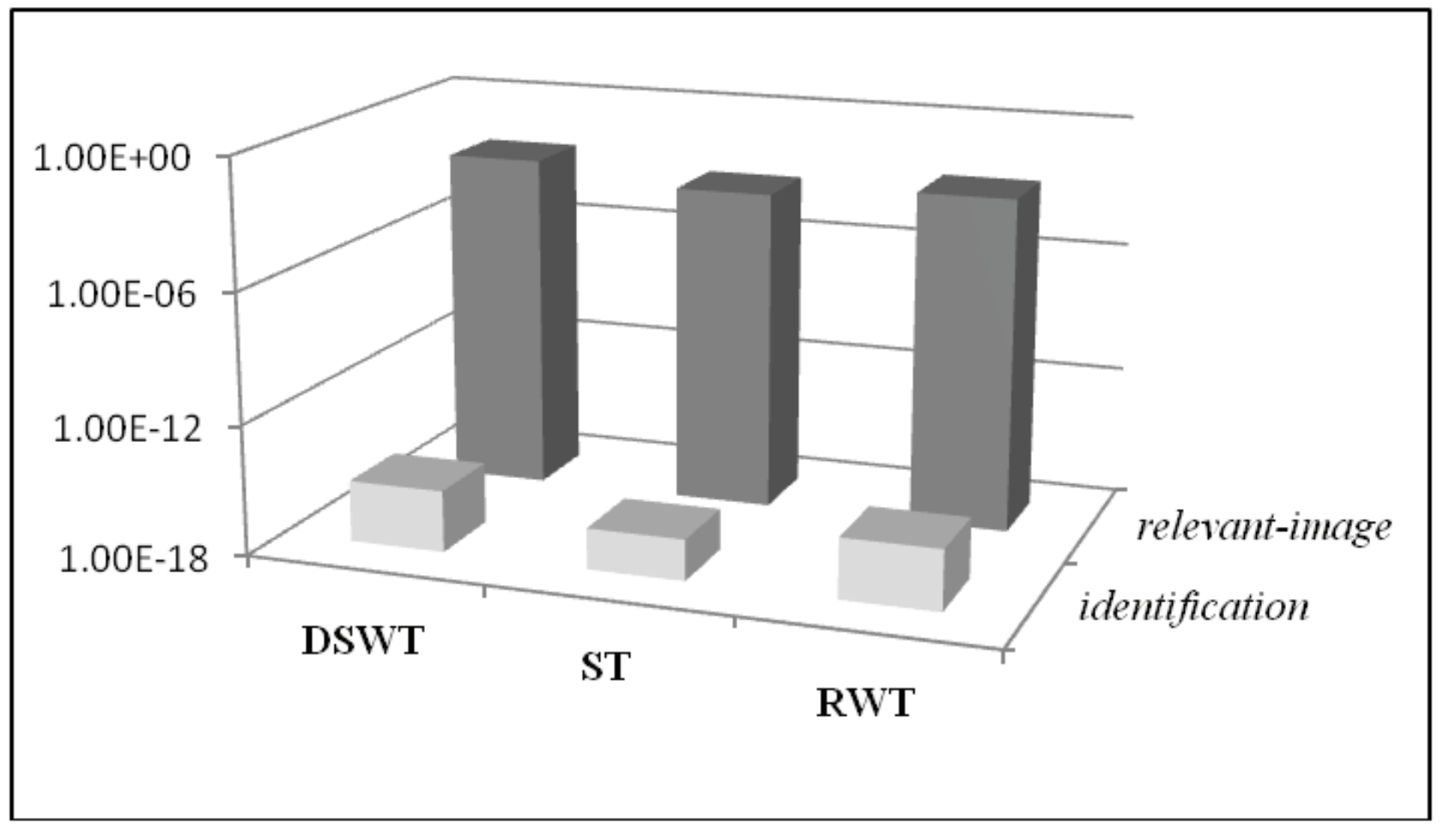

Figure 2. Similarity assessment in metrics 


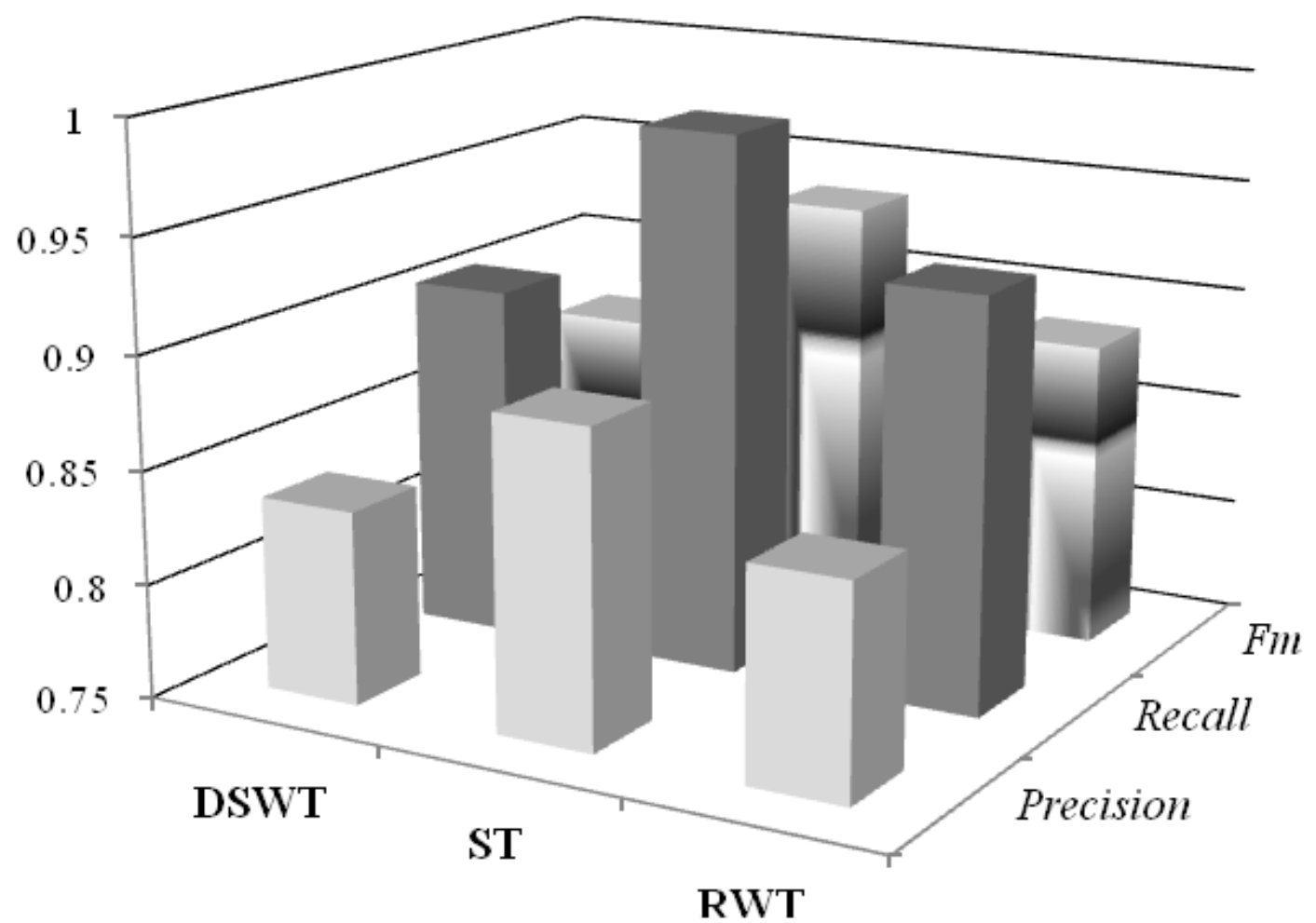

Figure 3. Metric assessments of the performance of the algorithms used

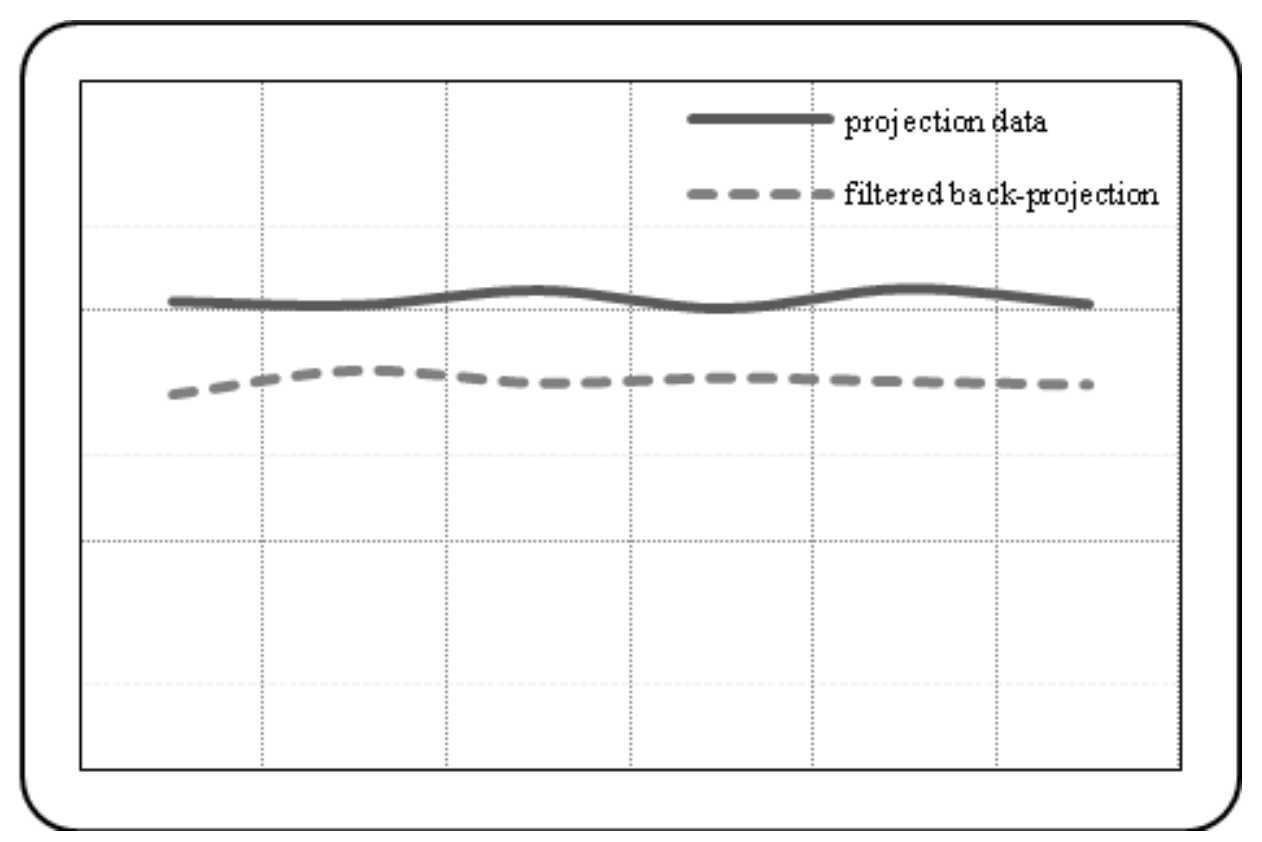

Figure 4. Graph of recognition metric values 
projection data in algorithms for retrieving images from a medical database when compared to using recovered tomographic slices.

From the conducted comparative analysis (in terms of precision, recall and $F_{m}$ ) it can be concluded that from the indexing multiscale methods that have been used the best results are obtained using Shearlet Transform.

Indexing the data recovered from the Radon space leads to loss of information which is essential in tasks of image recognition.

\section{References}

1. Gueild, M. O., M. Kohnen, D. Keysers, H. Schubert, B. Wien, J. Bredno, T. Leman. Quality of DICOM Header Information for Image Categorization. International Symposium on Medical Imaging,

\section{Manuscript received on 16.01.2016}

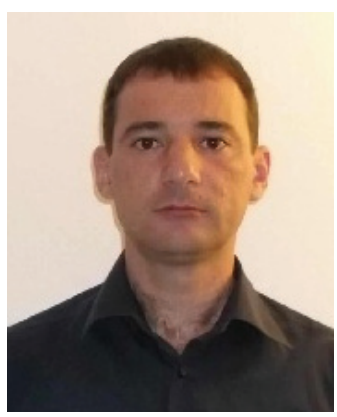

Assist. prof. Miroslav Petrov is graduated from Tehnical University of Varna. He recived $P h D$ degree from the Department of Computer Systems at the Tehnical University of Sofia. Since October 2005 he has worked as an assistant professor at the Department of Computer Systems and Technologies in the St. Cyril and St. Methodius University of Veliko Tarnovo. His current research interests are in the area of digital vision ,CBIR-systems. signal and image processing, computer

Contacts:

St. Cyril and St. Methodius University of Veliko Tarnovo Faculty of Mathematics and Informatics Department of Computer Systems and Technologies 3 Arh. G. Kozarov St., 5003 Veliko Tarnovo e-mail:m_petrov75@abv.bg
4685 of SPIE Proc., San Diego, CA, USA, 2002, 280-287.

2. Troitsky, I. Statistical Theory of Tomography. Moscow, Radio i Svyaz, 1989, ISBN 5-256-00182-5 (in Russian).

3. Mallat, S. A Wavelet Tour of Signal Processing. New York, Academic Press, 1998.

4. Kutyniok, G., D. Labate, Editors, Shearlets: Multiscale Analysis for Multivariate Data. Birkhäuser Basel (Springer Science+Business Media, LLC Birkhäuser Basel, 2012.

5. Häuser, S., G. Steidl. Fast Finite Shearlet Transform: a Tutorial. Preprint University of Kaiserslautern, 2014.

6. Lim, W. Q. The Discrete Shearlet Transform: a New Directional Transform and Compactly Supported Shearlet Frames. IEEE Trans. Imag. Proc. 19, 2010, 1166-1180.

7. Polqkova, M., V. Krylov. Morphologic Contour Method of Segmentation of Images Based on Repagulum Wavelet Transform. Proceedings of Odessa Polytechnic University, 1 (25), 2006 (in Russian). 